\title{
Trends in DNA barcoding and metabarcoding
}

\author{
Sarah J. Adamowicz, James S. Boatwright, Frédéric Chain, Brian L. Fisher, Ian D. Hogg, \\ Florian Leese, Darío A. Lijtmaer, Monica Mwale, Amanda M. Naaum, Xavier Pochon, \\ Dirk Steinke, John-James Wilson, Susanna Wood, Jianping Xu, Sen Xu, Xin Zhou, \\ and Michelle van der Bank
}

This open-access special issue features 12 full articles representing emerging trends from the international DNA barcoding community. Several articles highlight how DNA-based techniques are elucidating the species diversity, biogeography, and conservation status of Africa's biodiversity. Another prominent theme is the movement towards big biodiversity data using high-throughput, individual-based DNA barcoding methods, which preserve voucher specimens and abundance data, as well as bulk sample-based metabarcoding. Methodological developments are enhancing the detection of specific species and whole communities using environmental DNA (eDNA) barcoding and metabarcoding. Data are also expanding in terms of genetic coverage; in this issue, a new database is established for a secondary fungal DNA barcode marker, and multi-kingdom, multi-marker biodiversity surveys are gaining traction. DNA barcode sequence data, often combined with complementary markers or taxonomic information, are increasingly contributing to large-scale phylogenetic projects, with implications for understanding evolutionary history, community structure, and conservation priorities.

\section{Focus on African biodiversity}

This special issue of peer-reviewed papers focuses upon research trends and biological findings from the international DNA barcoding research community. Several contributions focus upon the unique biota of Africa. A number of African institutions, particularly in South Africa but elsewhere as well, harbour rich research programs in biodiversity, DNA barcoding, molecular phylogenetics, molecular wildlife forensics, and evolutionary community ecology (e.g., Adeoba et al. 2019; Yessoufou et al. 2019). Moreover, the 7th International Barcode of Life Conference was recently held at Kruger National Park, South Africa, from November 20-24, 2017, marking the first time this biennial conference series was hosted on the African continent (Adamowicz et al. 2017).

Key trends in contemporary DNA barcoding research include large-scale analyses of the biodiversity, evolutionary history, and conservation status of the biota of Africa; increasing use of DNA-based methods for wildlife forensics and for marketplace product authentication; cross-over of cutting-edge methods from genomics into biodiversity research; growing interest in the development and applications of environmental DNA (eDNA) techniques; and the integrative analysis of biomes and species interactions (Adamowicz et al. 2017). A strong signature of international collaboration and authorship is also a consistent pattern among researchers in the DNA barcoding com-

Received 19 March 2019. Accepted 19 March 2019.

S.J. Adamowicz and D. Steinke. University of Guelph, Guelph, Ontario, Canada.

J.S. Boatwright. University of the Western Cape, Cape Town, Western Cape, South Africa.

F. Chain. University of Massachusetts, Lowell, Massachusetts, USA.

B.L. Fisher. California Academy of Sciences, San Francisco, California, USA.

I.D. Hogg. Polar Knowledge Canada, Cambridge Bay, Nunavut, Canada.

F. Leese. University of Duisburg-Essen, North Rhine-Westphalia, Germany.

D.A. Lijtmaer. Museo Argentino de Ciencias Naturales "Bernardino Rivadavia" - CONICET, Buenos Aires, Argentina.

M. Mwale. South African National Biodiversity Institute, National Zoological Gardens, Pretoria, Gauteng, South Africa.

A.M. Naaum. TRU-ID Ltd., Guelph, Ontario, Canada; Institute for Global Food Security, School of Biological Sciences, Queen's

University Belfast, Belfast, Northern Ireland, United Kingdom.

X. Pochon. Cawthron Institute, Nelson, New Zealand \& Institute of Marine Science, University of Auckland, Auckland, New Zealand.

J.-J. Wilson. World Museum, National Museums Liverpool, Liverpool, United Kingdom.

S. Wood. Cawthron Institute, Nelson, New Zealand.

J. Xu. McMaster University, Hamilton, Ontario, Canada.

S. Xu. University of Texas at Arlington, Arlington, Texas, USA.

X. Zhou. Department of Entomology, China Agricultural University, Beijing, China, 100193.

M. van der Bank. University of Johannesburg, Johannesburg, Gauteng, South Africa.

Corresponding author: Sarah J. Adamowicz (email: sadamowi@uoguelph.ca).

Copyright remains with the author(s) or their institution(s). Permission for reuse (free in most cases) can be obtained from RightsLink. 
munity and reflects a global research network (Adamowicz and Steinke 2015; Adamowicz et al. 2017; also see references herein).

Here, our team of invited Guest and Regular Associate Editors is pleased to introduce a special issue of 12 openaccess articles that use or develop DNA barcoding and related techniques to further the study of biodiversity, evolution, and conservation. This will be followed by the publication of a larger virtual special issue, comprised of an array of papers from the 7th Conference plus additional related contributions published across multiple issues of Genome. In addition to a geographic focus on the biota of Africa in multiple contributions, this special issue showcases three main scientific trends.

\section{Large-scale biodiversity analyses}

A dominant trend in DNA barcoding research, exemplified in this special issue, is the development of new approaches for acquiring big biodiversity data. For example, deWaard et al. (2019) present protocols for efficient, high-throughput biodiversity surveys of arthropod samples, using a workflow that combines Malaise trap sampling, specimen sorting, DNA barcoding via unidirectional sequencing, grouping specimens into species-like units termed Barcode Index Numbers (BINs, Ratnasingham and Hebert 2013), selective photography of representative specimens from BINs, and selective bidirectional sequencing. This workflow preserves a match between each DNA sequence and its voucher specimen, thus building up a reference resource and resulting in abundance-based biodiversity data.

Two contributions to this special issue apply individualbased DNA barcoding methods to hyper-diverse and underexplored biotas. Focusing on an underexplored area of the Afrotropical biogeographic region, Delabye et al. (2019) survey and DNA barcode macromoths at two sites in Gabon. This team has discovered more provisional species than had been previously recorded in prior species lists for the entire country. This study uncovers a high biodiversity as well as high spatial and temporal turnover, with a preponderance of singleton BINs detected. DNA barcoding can help to overcome taxonomic impediments and enable researchers to quantify and compare biodiversity across sites in tropical biotas. On the biogeographically unique island of Madagascar, Lopez-Vaamonde et al. (2019) survey micromoths at several sites and find similarly high diversity and novelty, with $98 \%$ of BINs being new to BOLD (the Barcode of Life Data Systems; Ratnasingham and Hebert 2007) and a high proportion of singletons. With evidence of multiple invasions detected in disturbed habitats, their study showcases the unique biodiversity of Madagascar as well as the contribution that DNA-based methods can make to the study of endemism and distributional patterns, particularly when using standardized gene regions to enable comparison with sequences in public databases.
While individual-based methods provide abundance data, substantial research attention is also being directed to exploring prospects for more efficient biodiversity data collection. In this issue, Zizka et al. (2019) present a methodological study of biodiversity detection by eDNA metabarcoding from the fixative used to store bulk macroinvertebrate samples. By constructing known mock communities, they compare species recovery from the sample fixative against DNA barcoding of specimens as well as bulk sample metabarcoding. Their results are encouraging, showing in general good species recovery, albeit with variable DNA sequence read counts. The authors suggest that classical DNA barcoding or bulk metabarcoding may be preferred when a more comprehensive biodiversity survey is needed, yet eDNA metabarcoding from preservative is a promising alternative for large-scale, efficient, and cost-effective biodiversity studies.

The challenges of detecting species in the wild using molecular approaches are also highlighted by Wood et al. (2019), who compare surveys of marine environments conducted by SCUBA divers against results obtained using quantitative PCR (qPCR) performed on eDNA and eRNA. Their detection rates with qPCR are lower than for diver surveys and vary considerably between the two invasive species targeted for this study: a polychaete worm and a tunicate species. Nevertheless, the species are detected with qPCR at some sites where diver surveys could not be performed. These findings suggest the need for an understanding of DNA shedding rates among taxa as well as studies of the fate of eDNA in natural environments. The authors highlight eDNA as a complementary source of information, and their findings emphasize the value of methodological comparisons and validation studies to understand the potential strengths and limitations of eDNA-based methods.

\section{Multi-marker and holistic approaches to the study of biodiversity}

A second notable trend is towards increasingly holistic approaches to biodiversity analysis, jointly considering multiple kingdoms and scales of biodiversity. In this issue, Ritter et al. (2019) explore a multi-faceted approach to high-throughput bioinventory, focused more on capturing total biodiversity rather than on abundance data. They advocate for a combined sampling scheme, consisting of soil samples and insect trapping (such as through Malaise traps), followed by eDNA metabarcoding the soil biota as well as metabarcoding of DNA extracted from the bulk insect samples using a non-destructive protocol. A multi-marker metabarcoding approach was then used to survey both eukaryotic and prokaryotic components of biodiversity. The authors suggest a controlled approach to field sampling and note that the different collecting methods yield highly complementary biodiversity components. 
In addition to capturing more total biodiversity, multimarker approaches can also increase confidence in identifications within taxonomic groups. Meyer et al. (2019) promote a multi-marker approach for fungal DNA barcoding. They introduce a new database for the secondary DNA barcode marker, Translational Elongation Factor $1 \alpha$ (TEF1 $\alpha$ ), for fungi. Complementing the ITS marker, which was established in 2012 as the standard for fungi (Schoch et al. 2012), TEF1 $\alpha$ is proposed to increase resolution at the species level. Confidence in identifications is especially important in clinical settings when dealing with pathogenic species. Formal establishment of multiple markers mirrors the multi-marker approach for plant DNA barcoding and opens new potential avenues for DNA barcoding applications as well as fundamental research in biodiversity patterns and systematics.

\section{Tree of life and phylogenetic diversity}

A third research trend involves working towards more complete phylogenetic trees and using phylogenetic hypotheses to study ecological and evolutionary mechanisms. Methods for building phylogenetic trees vary, such as through de novo phylogenetic reconstruction using multi-marker datasets or hybrid taxonomic and phylogenetic approaches. By participating in large-scale biodiversity surveys and DNA sequencing campaigns, the international DNA barcoding research community is establishing new ways to fill in leaves on the tree of life and is achieving more comprehensive phylogenetic coverage than was considered possible a decade ago.

A notable example of this trend is this issue's contribution by Adeoba et al. (2019), who combine taxonomic and DNA barcode sequence data to create a new phylogenetic hypothesis for species belonging to the fish family Cyprinidae in Africa. They conclude that there is a significant phylogenetic signal in the distribution of heightened extinction risk, meaning that more unique evolutionary history would be lost than expected by chance alone should currently threatened species be permitted to go extinct. This pattern could cause the loss of unique ecosystem functions as well as inherently valuable, unique life forms. Adeoba et al. (2019) also highlight the identities and distributions of species of special relevance for conservation due to their global evolutionary uniqueness and threatened status. Their approach highlights the utility of building comprehensive phylogenetic hypotheses at the species level yet also draws attention to deficits in taxonomic, geographic, and genetic marker coverage in public biological data bases. Increasing coverage across these domains would open new research avenues in conservation phylogenetics and DNA-based biodiversity tracking, informing conservation prioritization efforts.

Taxonomic and phylogenetic approaches are also being used to elucidate biodiversity and ecological interactions among species of socio-economic importance. Powell et al. (2019) combine molecular and mor- phological approaches to estimate fruit fly infestation rates as well as the diversity and associations of parasitoid wasps in both wild and cultivated olives in South Africa. In their study, Powell and colleagues discovered concordance between the different lines of evidence and a new understanding of the diversity and parasitism rates in olives.

Phylogenetic methods applied to DNA barcodes are also being used to elucidate biogeographic history and evolutionary rates, particularly over recent geological time periods, because commonly used DNA barcode markers yield a phylogenetic signal at low taxonomic levels. Loeza-Quintana et al. (2019) generate phylogenetic hypotheses for trans-Arctic marine invertebrates and compare phylogeographic patterns against the timeline of the recurrent openings and closures of the Bering Strait. By arranging the biogeographic and geological/ climatic evidence in a harmonized timeline, they propose a recalibrated molecular clock for northern marine invertebrates. This research involving many allopatric sister pairs-and with molluscan fossil evidence used to validate the biogeography-based calibrations-has resulted in an upward revision of rates of molecular evolution in the mitochondrial cytochrome $c$ oxidase subunit I (COI) gene compared to commonly cited prior marine clock calibration studies. These findings are expected to inform rates of diversification as well as the correlation between evolutionary events and the geological and climatic history of marine regions.

There is also an increasing tendency in the DNA barcoding community, particularly among botanical researchers, to use DNA barcode loci to construct whole-community phylogenies. For Robben Island, South Africa, Yessoufou et al. (2019) generate a community-wide phylogenetic tree and reveal an association between higher phylogenetic diversity within native communities and lower risk of localscale invasion by non-native species. They also pinpoint differences in biological traits, such as flowering time, pollination syndrome, and breeding mode, between nonnative and native species. Their work contributes to an understanding of factors governing invasion processes and the importance of preserving phylogenetically diverse native plant communities.

Moving into the aquatic environmental microbiome, Farrell et al. (2019) conduct bacterial metabarcoding and phylogenetic analysis using the 16S rRNA gene on uncultured water samples from watering holes in Kruger National Park and found spatially and phylogenetically structured communities. This environmental microbiome approach could be paired in the future with multi-marker metabarcoding, including the macrobiota, to examine biological associations between the prokaryotes and eukaryotes and to track spatial and temporal changes. 


\section{Looking ahead}

The 7th Conference participants described increasing research interest in applying high-throughput sequencing technologies to various dimensions of biodiversity analysis. There is an apparent need for further controlled studies, including thoughtfully constructed mock communities consisting of both prokaryotic and eukaryotic components. Such analyses will help characterize the strengths and limitations of different biodiversity detection methods. New research could be productively targeted towards DNA shedding rates, spatial distribution and movement, and the degradation rates of eDNA in natural systems. The next phases of methodological development should also include comparative research into bioinformatic methods for processing high-throughput sequence data and combining DNA barcoding (many taxa, few genes) and phylogenomics (few taxa, many genetic regions) evidence into global phylogenies that can approach completeness at an unprecedented scale. This dialogue will continue amid more exciting discoveries in Trondheim, Norway, from June 17-20, 2019, during the 8th International Barcode of Life Conference.

\section{References}

Adamowicz, S.J., and Steinke, D. 2015. Increasing global participation in genetics research through DNA barcoding. Genome, 58(12): 519-526. doi:10.1139/gen-2015-0130. PMID:26642251.

Adamowicz, S.J., Hollingsworth, P.M., Ratnasingham, S., and van der Bank, M. 2017. International Barcode of Life: Focus on big biodiversity in South Africa. Genome, 60(11): 875-879. doi:10.1139/gen-2017-0210. PMID:29130757.

Adeoba, M.I., Tesfamichael, S., and Yessoufou, K. 2019. Preserving the tree of life of the fish family Cyprinidae in Africa in the face of the ongoing extinction crisis. Genome, 62. [This issue.] doi:10.1139/gen-2018-0023.

Delabye, S., Rougerie, R., Bayendi, S., Andeime-Eyene, M., Zakharov, E.V., deWaard, J.R., Hebert, P.D.N., Kamgang, R., Le Gall, P., Lopez-Vaamonde, C., Mavoungou, J.-F., Moussavou, G., Moulin, N., Oslisly, R., Rahola, N., Sebag, D., and Decaëns, T. 2019. Characterization and comparison of poorly known moth communities through DNA barcoding in two Afrotropical environments in Gabon. Genome, 62. [This issue.] doi:10.1139/gen-2018-0063.

deWaard, J.R., Levesque-Beaudin, V., deWaard, S.L., Ivanova, N.V., McKeown, J.T.A., Miskie, R., Naik, S., Perez, K.H.J., Ratnasingham, S., Sobel, C.N., Sones, J.E., Steinke, C., Telfer, A.C., Young, A.D., Young, M.R., Zakharov, E.V., and Hebert, P.D.N. 2019. Expedited assessment of terrestrial arthropod diversity by coupling Malaise traps with DNA barcoding. Genome, 62. [This issue.] doi:10.1139/gen-2018-0093.

Farrell, M.J., Govender, D., Hajibabaei, M., van der Bank, M., and Davies, T.J. 2019. Bacterial diversity in the waterholes of the Kruger National Park: an eDNA metabarcoding approach. Genome, 62. [This issue.] doi:10.1139/gen-2018-0064.

Loeza-Quintana, T., Carr, C.M., Khan, T., Bhatt, Y.A., Lyon, S.P., Hebert, P.D.N., and Adamowicz, S.J. 2019. Recalibrating the molecular clock for Arctic marine invertebrates based on DNA barcodes. Genome, 62. [This issue.] doi:10.1139/gen-20180107.

Lopez-Vaamonde, C., Sire, L., Rasmussen, B., Rougerie, R., Wieser, C., Ahamadi Allaoui, A., Minet, J., deWaard, J.R., Decaëns, T., and Lees, D.C. 2019. DNA barcodes reveal deeply neglected diversity and numerous invasions of micromoths in Madagascar. Genome, 62. [This issue.] doi:10.1139/gen-20180065.

Meyer, W., Irinyi, L., Thuy Vi Hoang, M., Robert, V., GarciaHermoso, D., Desnos-Ollivier, M., Yurayart, C., Tsang, C.-C., Lee, C.-Y., Woo, P.C.Y., Mikhailovich Pchelin, I., Uhrlaß, S., Nenoff, P., Chindamporn, A., Chen, S., Hebert, P.D.N., Sorrell, T.C., and the ISHAM barcoding of pathogenic fungi working group. 2019. Database establishment for the secondary fungal DNA barcode translational elongation factor $1 \alpha$ (TEF1 $\alpha$ ). Genome, 62. [This issue.] doi:10.1139/gen-2018-0083.

Powell, C., Caleca, V., Sinno, M., van Staden, M., van Noort, S., Rhode, C., Allsopp, E., and van Asch, B. 2019. Barcoding of parasitoid wasps (Braconidae and Chalcidoidea) associated with wild and cultivated olives in the Western Cape of South Africa. Genome, 62. [This issue.] doi:10.1139/gen-2018-0068.

Ratnasingham, S., and Hebert, P.D.N. 2007. BOLD: the barcode of life data system (http://www.barcodinglife.org). Mol. Ecol. Notes, 7: 355-364. doi:10.1111/j.1471-8286.2007.01678.x. PMID: 18784790.

Ratnasingham, S., and Hebert, P.D.N. 2013. A DNA-based registry for all animal species: the Barcode Index Number (BIN) system. PLoS ONE, 8(7): e66213. doi:10.1371/journal.pone.0066213. PMID: 23861743.

Ritter, C.D., Häggqvist, S., Karlsson, D., Sääksjärvi, I.E., Muasya, A.M., Nilsson, R.H., and Antonelli, A. 2019. Biodiversity assessments in the 21st century: the potential of insect traps to complement environmental samples for estimating eukaryotic and prokaryotic diversity using high-throughput DNA metabarcoding. Genome, 62. [This issue.] doi:10.1139/gen2018-0096.

Schoch, C.L., Seifert, K.A., Huhndorf, S., Robert, V., Spouge, J.L., Levesque, C.A., Chen, W., and Fungal Barcoding Consortium. 2012. Nuclear ribosomal internal transcribed spacer (ITS) region as a universal DNA barcode marker for Fungi. Proc. Natl. Acad. Sci. U.S.A. 109(16): 6241-6246. doi:10.1073/pnas.1117018109. PMID:22454494.

Wood, S.A., Pochon, X., Ming, W., von Ammon, U., Woods, C., Carter, M., Smith, M., Inglis, G., and Zaiko, A. 2019. Considerations for incorporating real-time PCR assays into routine marine biosecurity surveillance programmes: a case study targeting the Mediterranean fanworm (Sabella spallanzanii) and club tunicate (Styela clava). Genome, 62. [This issue.] doi: 10.1139/gen-2018-0021.

Yessoufou, K., Bezeng, B.S., Gaoue, O.G., Bengu, T., and van der Bank, M. 2019. Phylogenetically diverse native systems are more resistant to invasive plant species on Robben Island, South Africa. Genome, 62. [This issue.] doi:10.1139/gen2018-0039.

Zizka, V.M.A., Leese, F., Peinert, B., and Geiger, M.F. 2019. DNA metabarcoding from sample fixative as a quick and voucherpreserving biodiversity assessment method. Genome, 62. [This issue.] doi:10.1139/gen-2018-0048. 